\title{
A Critical Look at Growth Factors and Epithelial-to-Mesenchymal Transition in the Adult Kidney
}

\author{
Interrelationships between Growth Factors That Regulate EMT in the Adult Kidney
}

\author{
Nadia A. Wahab Roger M. Mason \\ Renal Section, Division of Medicine, Faculty of Medicine, Imperial College London, London, UK
}

\section{Key Words}

Epithelial-to-mesenchymal transition • Transforming growth factor $\beta$ - Connective tissue growth factor . Hepatocyte growth factor - Bone morphogenic protein-7

\begin{abstract}
In the adult kidney, the cellular phenotypes are maintained by a strict balance of growth factors. Epithelial-to-mesenchymal transition (EMT) is a program whereby injured epithelial cells that function as ion and fluid transporters become matrix remodelling mesenchymal cells. This process requires either transcriptional repression of genes that maintain the epithelial phenotype and transcriptional activation, or relieved repression of genes needed for functional myofibroblasts. The transcriptional regulators are controlled by several integrated signalling pathways which are triggered by growth factors. Emerging evidence indicates that the growth factors TGF $\beta / C T G F$ and BMP-7/HGF are the main determinants that maintain the two cellular phenotypes. Both TGF $\beta$ and BMP-7 counteract the activity of each other by cross-inducing their respective inhibitory Smads. Both growth factors may also induce the expression of other factors that can change the cellular environment and enhance their function. Chronic kidney diseases (regardless of the ae-
\end{abstract}

tiology of the disease) are associated with increased TGF $\beta$ and CTGF expression levels which, in turn, have an inverse effect on the activity level of BMP-7 and HGF, leading to an EMT of injured tubular epithelial cells and a progression of the disease. A detailed understanding of the complex interrelationship between these growth factors may lead to the development of novel drugs. Copyright $\odot 2006 \mathrm{~S}$. Karger AG, Basel

\section{Introduction}

Epithelial-to-mesenchymal transition (EMT) and mesenchymal-to-epithelial transition (MET), by switching on and off specific genes are important processes during early development [1]. In the adult kidney, cells which are derived from the metanephric mesenchyme appear to retain their plasticity [2], a feature which becomes important during the repair of an injured kidney. The transient appearance of myofibroblasts is essential to immediate repair following injury. However, their persistence is considered the major effector of renal fibrosis during chronic injury, including tubulointerstitial fibrosis (TIF) which is regarded as a common pathway leading to end-stage kidney failure [3]. While the role of myofibroblasts in TIF

\section{KARGER}

Fax +4161306 1234 E-Mail karger@karger.ch www.karger.com (c) 2006 S. Karger AG, Basel

$1660-2129 / 06 / 1044-0129 \$ 23.50 / 0$

Accessible online at:

www.karger.com/nee
Nadia Wahab

Renal Section, Division of Medicine

Faculty of Medicine, Imperial College London

London, W12 ONN (UK)

Tel. +44 208383 2718, Fax +44 208383 2062, E-Mail nadia.wahab@imperial.ac.uk 
is widely accepted, their precise origin and fate is still debated $[4,5]$. It has been suggested that they may be derived from a heterogeneous origin such as resident fibroblasts, bone marrow, circulating fibrocytes, perivascular smooth muscle cells or local EMT. EMT is also thought to cause the tubular atrophy associated with TIF [6]. The process of EMT was first demonstrated by Strutz et al. [7] and confirmed by the study of Iwano et al. [6] using genetic tagging of renal tubules in mice. However, the in vivo evidence of EMT as the major mediator of the pathogenesis and progression of TIF in chronic kidney diseases is not yet conclusive [8-10].

\section{Events Occurring during EMT}

EMT is a program whereby epithelial cells that function as ion and fluid transporters become matrix remodelling cells. Thus cells: (1) Lose their epithelial polarity, cellular adhesion molecules, cell-cell and cell-matrix contacts. (2) Reorganize their actin cytoskeleton from a cortical bundle formation that supported adhesion molecules into stress fibres containing de novo expressed $\alpha$-smooth muscle actin ( $\alpha$-SMA) that support migration and matrix remodelling; cells also exchange their cytokeratin intermediate filaments for a vimentin profile. (3) Express S100A4 that interacts with actin and myosin heavy chain IIA to enhance cell motility. (4) Disrupt the tubular basement membrane (TBM) and migrate into the interstitium where they synthesise increasing amounts of extracellular matrix (ECM) [11].

\section{Regulators of EMT}

Numerous in vitro studies have shown that EMT is regulated by growth factors, cytokines, metalloproteinases (MMPs) which can disrupt the TBM integrity [12, 13 ] and alteration in the TBM composition [12]. Currently, transforming growth factor (TGF) $\beta_{1}$ is the main growth factor reported to mediate the initiation and maintenance of the EMT process. The role and the possible molecular mechanisms whereby TGF $\beta$ functions were recently reviewed extensively by Zavadil and Bottinger [5]. Thus the present article will focus on the interrelationship between TGF $\beta$ and other growth factors reported to be involved in EMT (fig. 1).

The EMT process requires either transcriptional repression of genes that maintain the epithelial phenotype (e.g. E-cadherin, claudins, occludins, desmoplakin, des- moglein, $\alpha_{6} \beta_{4}$ integrins) and transcriptional activation, or relieved repression of genes needed for functional myofibroblasts (e.g. FN-EDA ${ }^{+}$, vimentin, $\alpha$-SMA). This occurs via induction of several transcription repressors that recognize specific motifs in the target promoters (e.g. snail/Slug, SIP1, Twist), via co-repressors that interact with and inactivate transcriptional factors (e.g. TGIF and SnoN) or via regulation of the level of inhibitors of differentiation (Id proteins) which can act as positive regulators of cell proliferation and negative regulators of cell differentiation [14]. These transcriptional regulators are under the control of several integrated signalling pathways including Smads, ERK MAPK, JNK, p38 MAPK, PI3K, NFкB and the Rho/ROCK pathways which can be triggered by TGF $\beta[5]$ and other growth factors.

\section{Growth Factors Which Mediate TGF $\beta$ Action}

Connective tissue growth factor (CTGF) is an immediate-early product and a master mediator of the profibrotic actions of TGF $\beta$ [15-17]. It promotes fibroblast proliferation, differentiation and ECM production [18]. In vitro, CTGF is rapidly induced in renal tubular epithelial cells (TECs) by TGF $\beta$ in a Smad3/Smad4-dependent manner [19]. Elevated expression of CTGF is also reported in TECs and interstitial cells at the sites of chronic interstitial damage [20]. Moreover, CTGF was identified among the genes upregulated in the unilateral ureteral obstruction (UUO) animal model of TIF, using Affymetrix gene microarrays [21]. The crucial role of CTGF in mediating EMT in vitro was demonstrated by its direct ability to induce the process when added exogenously to TECs, and by the blockade of TGF $\beta$-induced EMT when CTGF antisense oligodeoxynucleotides (CTGF-AO) were used to deplete endogenous CTGF in TGF $\beta$-stimulated cells [22]. Moreover, administration of CTGF-AO attenuated the induction of CTGF, FN, FN-EDA ${ }^{+}, \alpha 1$ (type I) collagen and $\alpha$-SMA genes as well as the interstitial fibrotic areas in the UUO model, despite the elevated TGF $\beta$ gene expression which was not affected [20]. Similarly, Okada et al. [23] have shown that administration of CTGF-AO blocked CTGF expression in the TECs of the remnant kidney of TGF $\beta_{1}$-transgenic mice, which were subtotally nephrectomized. Again, in these experiments a sustained elevation of TGF $\beta_{1}$ mRNA was observed, despite the significant suppression of TIF in these animals. These findings indicate that CTGF is likely to have a direct effect on inducing EMT in vivo. 


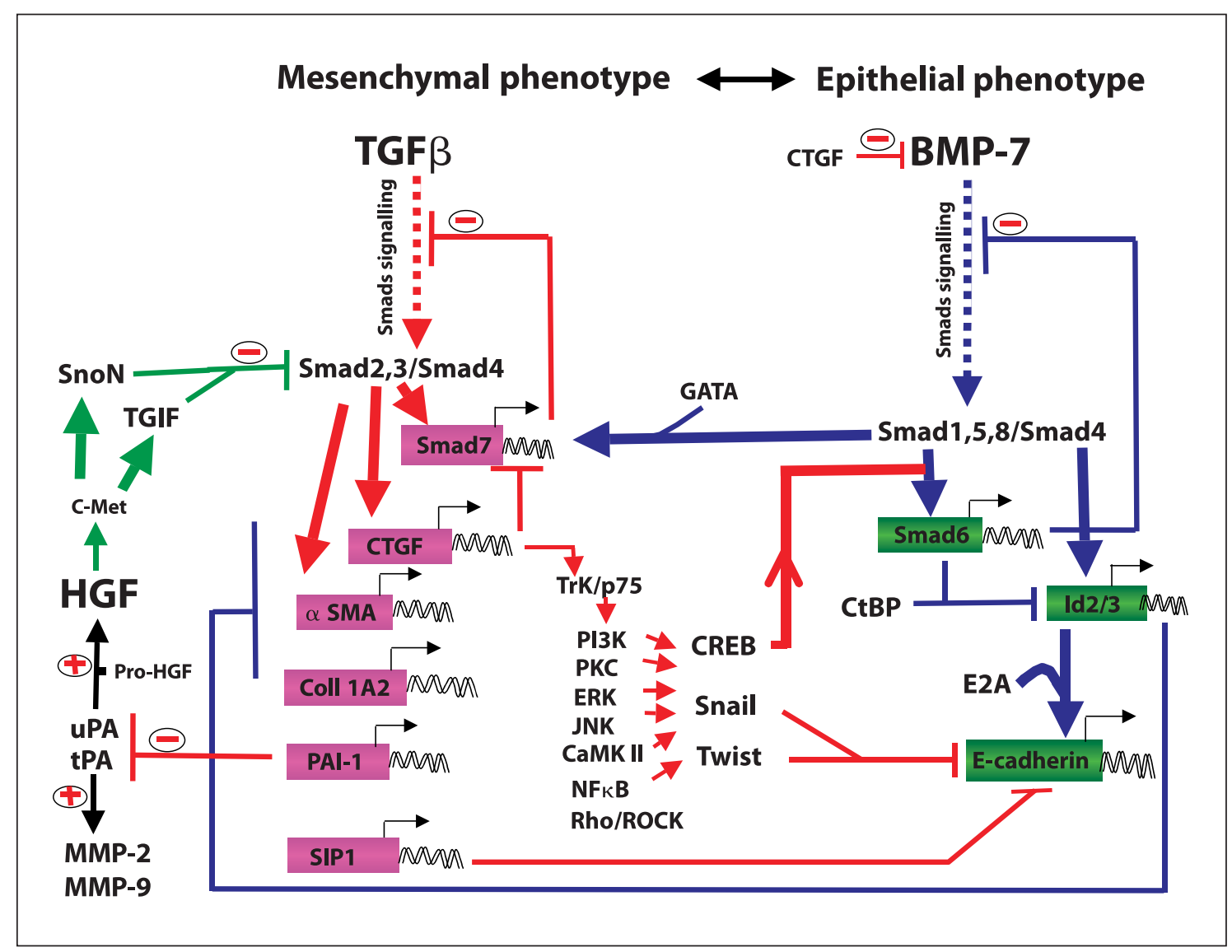

Fig. 1. Complex pattern of interaction between growth factors TGFß/CTGF and BMP-7/HGF. BMP-7 supports the epithelial phenotype by inducing the expression of Smad7 which inhibits TGF $\beta$ signalling, and Id $2 / 3$ which inactivate the repressor E2A to permit the expression of E-cadherin. Ids also inhibit several TGF $\beta$ responsive genes including CTGF. HGF also supports the same phenotype by inducing SnoN and TGIF which inactivate the Smad2/3/4 complex in the nucleus and repress the transcription of several TGF $\beta$-responsive genes, including CTGF and $\alpha$-SMA. On the other hand, TGF $\beta$ supports the mesenchymal phenotype by the rapid induction of CTGF. CTGF inhibits BMPs extracellularly, activates several receptor systems including $\mathrm{Trk} / \mathrm{p} 75^{\mathrm{NTR}}$ and triggers signalling pathways that integrate with TGF $3 /$ Smad signalling, leading to the induction of transcription repressors that inhibit E-cadherin. CTGF also inhibits the expression level of Smad7 thus enhancing the transcription of TGF $\beta$-responsive genes including $\alpha$-SMA and PAI-1. Moreover CREB, activated via CTGF, associates with the BMP/Smad complex to induce the expression of Smad6 which not only inhibits the BMP-7 signalling pathway but also the activity of Id2/3, leading to repression of Ecadherin and relieved repression from TGF $\beta$-responsive genes. TGF $\beta$-induced PAI-1 in turn inhibits the activities of uPA and tPA which can activate HGF and the MMPs.

\section{Growth Factors Which Contribute to TGF $\beta$ Action}

It has been reported that epidermal growth factor, basic fibroblast growth factor and platelet-derived growth factor-BB can induce EMT in vitro, but to a lesser extent than TGF $\beta$. However, they exhibit an enhancer effect when added with TGF $\beta_{1}$ to promote EMT [24-26]. These growth factors are known to signal via tyrosine kinase receptors that activate the same intracellular signalling pathways which integrate with the Smad pathway. Ex- pression of basic fibroblast growth factor is elevated in injured TECs and is thought to contribute to EMT during renal damage in immune-mediated injury $[25,27]$, mainly by increasing the level of MMP-2 and MMP-9 secretion, essential for the disruption of TBM. The inflammatory cytokine tumor necrosis factor- $\alpha$ produced by macrophages is also thought to accelerate TGF $\beta$-induced EMT [28] via the activation of p38 MAPK and NFкB transcription factors. 


\section{Growth Factors Which Induce TGF $\beta$}

Angiotensin II has been reported to induce the expression of TGF $\beta$ and CTGF [29] and is thus able to promote EMT in TECs [30]. Other factors such as interleukin (IL)1 and IL- 8 induce EMT via a TGF $\beta$-dependent pathway [31] but evidence that oncostatin $M$ does so in a similar manner is so far lacking [32]. Other EMT inducers such as advanced glycation end products have been reported to act through both TGF $\beta$-dependent and independent pathways [33].

\section{Growth Factors Which Antagonize TGF $\beta$ Action}

Hepatocyte growth factor (HGF) has been shown to completely block TGF $\beta$-induced EMT, to enhance matrix degradation in vitro and to reverse established TIF in animal models of chronic renal injury by inhibiting the TGF $\beta$ function [34]. The mechanism underlying this ability is thought to involve the induction of the Smad transcriptional co-repressors SnoN and TGIF, which in turn interact with and inactivate the activated Smad complexes, thereby repressing the transcription of several TGF $\beta$-responsive genes, including CTGF and $\alpha$-SMA $[35,36]$. HGF administration is reported to restore the level of SnoN and TGIF protein expression which are downregulated in the kidney of animal models of chronic renal injury.

Bone morphogenic protein-7 (BMP-7) is an essential growth factor during kidney development as it regulates branching morphogenesis via MET [37]. In the adult kidney, BMP-7 maintains the function of the renal epithelium. Acute and chronic tubular injury is associated with the downregulation of BMP-7 expression [38]. Recombinant BMP-7 has been shown to reverse TGF $\beta$-induced EMT in cultured TECs, and its administration led to the repair of severely damaged renal TECs and to improved renal function in animal models of chronic renal injury and fibrosis [39]. The underlying mechanism is thought to involve the induction of Id proteins by BMP-7 [40]. This is inhibited by TGF $\beta$ which promotes EMT. Ids lack a basic DNA binding region, but they possess a HLH dimerization motif which allows them to interact with and inactivate bHLH transcription factors that can be inhibitors or activators of transcription. CTGF, plasminogen activator inhibitor-1 (PAI-1) and thrombospondin-1 are among those TGF $\beta$ responsive genes that are directly downregulated by BMP-7 [41]. The blocking of TGF $\beta$ dependent upregulation of PAI-1 by BMP-7 also results in an induced expression of active MMP-2, which promotes the degradation of the fibrotic matrix [38]. This blocking may also enhance the activity of the urokinasetype plasminogen activator, a known activator of HGF $[42,43]$.

\section{Smad6 and Smad7 as Regulators of EMT}

TGF $\beta$ and BMP-7 signalling pathways are tightly regulated through both positive and negative mechanisms. One of these negative feedback mechanisms is the production of inhibitory Smads (Smad6/7). Whereas Smad6 preferentially inhibits BMP signalling, Smad7 inhibits the TGF $\beta$ /activin signalling pathway [44].

Smad7 binds E3-ubiquitin ligases of the smurf family which cause ubiquitination and proteasomal degradation of the TGF $\beta$ receptors [45] and prevents the phosphorylation of Smad 2 and Smad3, providing a negative feedback loop mechanism to shut off the TGF $\beta$ signal and limit its effects [46]. Reduction of the Smad7 expression level has been reported in the UUO animal model of TIF [47], while its forced expression blocks TGF $\beta$-induced EMT and ECM synthesis [48]. Smad7 is an immediate-early target gene for both TGF $\beta$ and BMP-7. While TGF $\beta$ induces its expression via a Smad binding element site that is regulated by Smad 3/4, AP-1, SP1, and TFE3; BMP-7 induces its expression via multiple BMP responsive elements (BRE). Two low-affinity sites, BRE-1/2, are activated at high concentrations of BMP and 1 high-affinity site, I-BRE, is also activated at low BMP concentrations. BRE-1 binds the Smad1/4 complex whereas I-BRE binds the Smad1/4/GATA complex [44]. This suggests that the presence of GATA transcription factors may enhance Smad7 induction leading to a blockade of TGF $\beta$ signalling and allowing BMP to signal even at low concentrations.

Smad6 is an immediate-early gene product of BMP signalling. However, its expression requires the cooperative association between the transcription factor CREB and the Smad1/5/4 complex [49]. Smad6 does not only inhibit the activation of R-Smads (Smad1/5/8) and the heteromerization of R-Smad-Smad4, but also acts directly as a transcriptional (co)repressor [50, 51]. Interestingly, it has been reported that Smad6 represses BMP-induced Ids transcription through recruiting the transcriptional corepressor C-terminal binding protein [52]. This indicates that induction of Smad6 may promote TGF $\beta$ to induce EMT. 


\section{Interrelationship between Growth Factors}

The inverse effects of TGF $\beta / C T G F$ and BMP-7/HGF on the EMT process suggest that these growth factors are the main determinants that maintain the two cellular phenotypes, with CTGF acting as a brake to control the level of Smad7 [16] and allow the TGF $\beta$ signalling pathway. CTGF also rapidly activates all the intracellular signalling pathways known to cross-talk with the Smad signalling pathway via the activation of several receptor systems including TrkA/p75 ${ }^{\mathrm{NTR}}$, integrins and LRP-2 [5355]. Preliminary results indicate that CTGF also activates the TrkA receptor in tubular cells in vivo in a CTGFoverexpressing transgenic mouse [Wahab and Mason unpubl. results]. CTGF can also inhibit the BMPs-Smad signalling pathway by binding BMPs extracellularly [56] . Both TGF $\beta$ and BMP-7 counteract the activity of each other by cross-inducing their respective inhibitory Smads. Both growth factors may also induce the expression of other factors that can change the cellular environment and enhance their function. Figure 1 represents a model to illustrate this complex pattern of interaction.

\section{Conclusion}

In the adult kidney, the cellular phenotypes are maintained by a strict balance of growth factors. Chronic kidney diseases (regardless of the aetiology of the disease) are associated with increased TGF $\beta$ and CTGF expression levels which in turn have an inverse effect on the activity level of BMP-7 and HGF, leading to EMT of injured TECs and progression of chronic kidney diseases. Removal of injury, reduction in TGF $\beta / C T G F$ levels or increase in BMP7/HGF levels may lead to regression of these diseases. These growth factors already represent targets for therapeutic intervention in the treatment of renal diseases, but a more detailed understanding of the complex processes involved may lead to the further development of novel drugs.

\section{References}

$\checkmark 1$ Boyer B, Thiery JP: Epithelium-mesenchyme interconversion as example of epithelial plasticity. APMIS 1993;101:257-268.

$\checkmark 2$ Horster MF, Braun GS, Huber SM: Embryonic renal epithelia: induction, nephrogenesis, and cell differentiation. Physiol Rev 1999;79:1157-1191.

$\checkmark 3$ Nath KA: The tubulointerstitium in progressive renal disease. Kidney Int 1998;54:992994.

4 Liu Y: Epithelial to mesenchymal transition in renal fibrogenesis: pathologic significance, molecular mechanism, and therapeutic intervention. J Am Soc Nephrol 2004;15: $1-12$.

$\checkmark 5$ Zavadil J, Bottinger EP: TGF-beta and epithelial-to-mesenchymal transitions. Oncogene 2005;24:5764-5774.

6 Iwano M, Plieth D, Danoff TM, et al: Evidence that fibroblasts derive from epithelium during tissue fibrosis. J Clin Invest 2002; 110:341-350.

7 Strutz F, Okada H, Lo CW, et al: Identification and characterization of a fibroblast marker: FSP1. J Cell Biol 1995;130:393-405.

$\checkmark 8$ Rastaldi MP, Ferrario F, Giardino L, et al: Epithelial-mesenchymal transition of tubular epithelial cells in human renal biopsies. Kidney Int 2002;62:137-146.

-9 Jinde K, Nikolic-Paterson DJ, et al: Tubular phenotypic change in progressive tubulointerstitial fibrosis in human glomerulonephritis. Am J Kidney Dis 2001;38:761-769.
10 Vongwiwatana A, Tasanarong A, Rayner DC, et al: Epithelial to mesenchymal transition during late deterioration of human kidney transplants: the role of tubular cells in fibrogenesis. Am J Transplant. 2005;5:13671374.

11 Yang J, Liu Y: Dissection of key events in tubular epithelial to myofibroblast transition and its implications in renal interstitial fibrosis. Am J Pathol 2001;159:1465-1475.

12 Zeisberg M, Bonner G, Maeshima Y, et al: Renal fibrosis: collagen composition and assembly regulates epithelial-mesenchymal transdifferentiation. Am J Pathol 2001;159: 1313-1321.

13 Cheng S, Lovett DH: Gelatinase A (MMP-2) is necessary and sufficient for renal tubular cell epithelial-mesenchymal transformation. Am J Pathol 2003;162:1937-1949.

14 Yokota Y, Mori S: Role of Id family proteins in growth control. J Cell Physiol 2002;190: 21-28.

15 Grotendorst GR, Okochi H, Hayashi N: A novel transforming growth factor beta response element controls the expression of the connective tissue growth factor gene. Cell Growth Differ 1996;7:469-480.

16 Wahab NA, Mason RM: Connective tissue growth factor and renal diseases: some answers, more questions. Curr Opin Nephrol Hypertens 2004;13:53-58.

17 Wahab NA, Weston BS, Mason RM: Modulation of the TGFbeta/Smad signaling pathway in mesangial cells by CTGF/CCN2. Exp Cell Res 2005;307:305-314.
18 Grotendorst GR, Rahmanie H, Duncan MR: Combinatorial signaling pathways determine fibroblast proliferation and myofibroblast differentiation. FASEB J 2004;18:469_479.

19 Phanish MK, Wahab NA, Colville-Nash P, et al: The differential role of Smad2 and Smad3 in the regulation of pro-fibrotic TGFbeta1 responses in human proximal-tubule epithelial cells. Biochem J 2006;393:601-607.

20 Yokoi H, Mukoyama M, Nagae T, et al: Reduction in connective tissue growth factor by antisense treatment ameliorates renal tubulointerstitial fibrosis. J Am Soc Nephrol 2004;15:1430-1440.

21 Higgins DF, Lappin DW, Kieran NE, et al: DNA oligonucleotide microarray technology identifies fisp-12 among other potential fibrogenic genes following murine unilateral ureteral obstruction (UUO): modulation during epithelial-mesenchymal transition. Kidney Int 2003;64:2079-2091.

22 Zhang C, Meng X, Zhu Z, et al: Connective tissue growth factor regulates the key events in tubular epithelial to myofibroblast transition in vitro. Cell Biol Int 2004;28:863-873.

$>23$ Okada H, Kikuta T, Kobayashi T, et al: Connective tissue growth factor expressed in tubular epithelium plays a pivotal role in renal fibrogenesis. J Am Soc Nephrol 2005;16: 133-143. 
-24 Terzi F, Burtin M, Hekmati M, et al: Targeted expression of a dominant-negative EGF-R in the kidney reduces tubulo-interstitial lesions after renal injury. J Clin Invest 2000;106: 225-234.

-25 Strutz F, Zeisberg M, Ziyadeh FN, et al: Role of basic fibroblast growth factor-2 in epithelial-mesenchymal transformation. Kidney Int 2002;6:1714-1728.

-26 Yamate J, Kuribayashi M, Kuwamura M, et al: Differential immunoexpressions of cytoskeletons in renal epithelial and interstitial cells in rat and canine fibrotic kidneys, and in kidney-related cell lines under fibrogenic stimuli. Exp Toxicol Pathol 2005;57:135147.

-27 Strutz F, Zeisberg M, Hemmerlein B, et al: Basic fibroblast growth factor expression is increased in human renal fibrogenesis and may mediate autocrine fibroblast proliferation. Kidney Int 2000;57:1521-1538.

-28 Bates RC, Mercurio AM: Tumor necrosis factor-alpha stimulates the epithelial-tomesenchymal transition of human colonic organoids. Mol Biol Cell 2003;14:17901800.

$\checkmark 29$ Rodriguez-Vita J, Sanchez-Lopez E, Esteban V, et al: Angiotensin II activates the Smad pathway in vascular smooth muscle cells by a transforming growth factor-beta-independent mechanism. Circulation 2005;111: 2509-2517.

-30 Yang J, Dai C, Liu Y: Hepatocyte growth factor gene therapy and angiotensin II blockade synergistically attenuate renal interstitial fibrosis in mice. J Am Soc Nephrol 2002;13: 2464-2477.

-31 Fan JM, Huang XR, Ng YY, et al: Interleukin1 induces tubular epithelial-myofibroblast transdifferentiation through a transforming growth factor-beta1-dependent mechanism in vitro. Am J Kidney Dis 2001;37:820-831.

>32 Nightingale J, Patel S, Suzuki N, et al: Oncostatin $\mathrm{M}$, a cytokine released by activated mononuclear cells, induces epithelial cellmyofibroblast transdifferentiation via Jak/ Stat pathway activation. J Am Soc Nephrol 2004;15:21-32.

>33 Li JH, Wang W, Huang XR, et al: Advanced glycation end products induce tubular epithelial-myofibroblast transition through the RAGE-ERK1/2 MAP kinase signaling pathway. Am J Pathol 2004;164:1389-1397.

-34 Yang J, Dai C, Liu Y: A novel mechanism by which hepatocyte growth factor blocks tubular epithelial to mesenchymal transition. J Am Soc Nephrol 2005;16:68-78.
35 Inoue T, Okada H, Kobayashi T, et al: Hepatocyte growth factor counteracts transforming growth factor-betal, through attenuation of connective tissue growth factor induction, and prevents renal fibrogenesis in 5/6 nephrectomized mice. FASEB J 2003;17: 268-270.

36 Dai C, Liu Y: Hepatocyte growth factor antagonizes the profibrotic action of TGFbeta1 in mesangial cells by stabilizing Smad transcriptional corepressor TGIF. J Am Soc Nephrol 2004;15:1402-1412.

37 Vukicevic S, Kopp JB, Luyten FP, et al: Induction of nephrogenic mesenchyme by osteogenic protein 1 (bone morphogenetic protein 7). Proc Natl Acad Sci USA 1996;93: 9021-9026.

38 Zeisberg M, Hanai J, Sugimoto H, et al: BMP7 counteracts TGF-beta1-induced epithelialto-mesenchymal transition and reverses chronic renal injury. Nat Med 2003;9:964968.

39 Zeisberg M, Bottiglio C, Kumar N, et al: Bone morphogenic protein-7 inhibits progression of chronic renal fibrosis associated with two genetic mouse models. Am J Physiol Renal Physiol 2003;285:F1060-F1067.

40 Kowanetz M, Valcourt U, Bergstrom R: Id2 and Id 3 define the potency of cell proliferation and differentiation responses to transforming growth factor beta and bone morphogenetic protein. Mol Cell Biol 2004;24: 4241-4254.

41 Wang S, Hirschberg R: Bone morphogenetic protein-7 signals opposing transforming growth factor beta in mesangial cells. J Biol Chem 2004;279:23200-23206.

42 Naldini L, Vigna E, Bardelli A, et al: Biological activation of pro-HGF (hepatocyte growth factor) by urokinase is controlled by a stoichiometric reaction. J Biol Chem 1995; 270:603-611.

43 Zeisberg M, Shah AA, Kalluri R: Bone morphogenic protein-7 induces mesenchymal to epithelial transition in adult renal fibroblasts and facilitates regeneration of injured kidney. J Biol Chem 2005;280:8094-8100.

44 Benchabane H, Wrana JL: GATA- and Smad1-dependent enhancers in the Smad7 gene differentially interpret bone morphogenetic protein concentrations. Mol Cell Biol 2003;23:6646-6661.

45 Ebisawa T, Fukuchi M, Murakami G, Chiba $\mathrm{T}$, Tanaka K, Imamura $\mathrm{T}$, Miyazono $\mathrm{K}$ : Smurf1 interacts with transforming growth factor-beta type I receptor through Smad7 and induces receptor degradation. J Biol Chem 2001;276:12477-12480.
46 Ulloa L, Doody J, Massague J: Inhibition of transforming growth factor-beta/SMAD signalling by the interferon-gamma/STAT pathway. Nature 1999;397:710-713.

47 Fukasawa H, Yamamoto T, Togawa A, et al: Down-regulation of Smad7 expression by ubiquitin-dependent degradation contributes to renal fibrosis in obstructive nephropathy in mice. Proc Natl Acad Sci USA. 2004; 101:8687-8692.

48 Li JH, Zhu HJ, Huang XR, Lai KN, Johnson RJ, Lan HY: Smad7 inhibits fibrotic effect of TGF-Beta on renal tubular epithelial cells by blocking Smad 2 activation. J Am Soc Nephrol 2002;13:1464-1472.

49 Ionescu AM, Drissi H, Schwarz Em, et al: CREB Cooperates with BMP-stimulated Smad signaling to enhance transcription of the Smad6 promoter. J Cell Physiol 2004;198: 428-440.

50 Bai S, Shi X, Yang X, et al: Smad6 as a transcriptional corepressor. J Biol Chem 2000; 275:8267-8270.

51 Hata A, Lagna G, Massague J, et al: Smad6 inhibits BMP/Smad1 signaling by specifically competing with the Smad4 tumor suppressor. Genes Dev 1998;12:186-197.

52 Lin X, Liang YY, Sun B, et al: Smad6 recruits transcription corepressor $\mathrm{CtBP}$ to repress bone morphogenetic protein-induced transcription. Mol Cell Biol 2003;23:9081-9093.

53 Wahab NA, Weston BS, Mason RM: Connective tissue growth factor CCN2 interacts with and activates the tyrosine kinase receptor TrkA. J Am Soc Nephrol 2005;16:340351.

54 Segarini PR, Nesbitt JE, Li D, Hays LG, Yates JR 3rd, Carmichael DF: The low density lipoprotein receptor-related protein/alpha2macroglobulin receptor is a receptor for connective tissue growth factor. J Biol Chem 2001;276:40659-40667.

55 Babic AM, Chen CC, Lau LF: Fisp12/mouse connective tissue growth factor mediates endothelial cell adhesion and migration through integrin alphavbeta3, promotes endothelial cell survival, and induces angiogenesis in vivo. Mol Cell Biol 1999;19:29582966.

56 Abreu JG, Ketpura NI, Reversade B, et al: Connective-tissue growth factor (CTGF) modulates cell signalling by BMP and TGFbeta. Nat Cell Biol 2002;4:599-604. 